\title{
The Simulation Course: An Innovative Way of Teaching Computational Science in Aeronautics
}

\author{
Ricard González-Cinca ${ }^{1}$, Eduard Santamaria ${ }^{2}$, and J. Luis A. Yebra ${ }^{3}$ \\ ${ }^{1}$ Department of Applied Physics \\ ${ }^{2}$ Department of Computer Architecture \\ ${ }^{3}$ Department of Applied Mathematics IV \\ Castelldefels School of Technology (EPSC) \\ Technical University of Catalonia (UPC) \\ Av. del Canal Olímpic, 15 - 08860 Castelldefels, Spain \\ ricard@fa.upc.edu
}

\begin{abstract}
This article describes an innovative methodology for teaching an undergraduate course on Computational Science, with a particular emphasis in Computational Fluid Dynamics (CFD), and the experiences derived from its implementation. The main activities taking place during this course are: development by students of a training project on a topic in materials science, development of a larger CFD project, and an introduction to a CFD commercial package. Projects are carried out by groups of students and are assigned from a set of different available possibilities. Project development consists in the implementation in code of the corresponding mathematical models and a graphical interface which permits the visualization of the results derived from the numerical resolution of the models. The main innovative aspects of the methodology are the use of Project Based Learning combined with the participation of lecturers from different areas of expertise. Other innovative issues include the opportunity for students to practice skills such as report writing, doing oral presentations, the use of English (a foreign language for them) and the use of Linux as the development environment.
\end{abstract}

\section{Introduction}

The Simulation course belongs to the bachelor's degree in Aeronautical Engineering (Air Navigation specialization) offered at the Technical School of Castelldefels (EPSC) of the Technical University of Catalonia (UPC). The bachelor's degree in Aeronautical Engineering started in 2002 and Simulation is being run since 2004. It is an elective course which students can enrol during the third year of their studies. The course complements previous training which mainly focuses on Air Navigation topics. Simulation has always been developed with the idea of applying an innovative methodology for teaching Computational Science, with a particular emphasis in CFD.

The broad context in which Simulation is being developed requires an introduction in order to better understand its innovative aspects. Spanish higher

M. Bubak et al. (Eds.): ICCS 2008, Part II, LNCS 5102, pp. $735-7442008$.

(C) Springer-Verlag Berlin Heidelberg 2008 
education system is currently in an adaptation process to the new European framework. Technical universities are composed by schools or faculties and departments. In the still active system schools have the role of offering degrees which last either three years ('technical engineering' or bachelor degree) or five years ('higher engineering' degree or bachelor + master degree). The unifying characteristics of the members of a department is their area of expertise, that is the scientific background and research interests (e.g. physics or mathematics). Courses in the degrees curricula are assigned to the department which has the closest area of expertise to the course. Although several courses have characteristics shared by different areas, in Spanish universities they use to be assigned to just one department; in other words, it is very unlikely that lecturers from different departments participate in the same course.

The two main features of the innovative methodology presented in this paper are the use of Project Based Learning [123] and the participation of lecturers from three main areas: physics, mathematics and computation. In Section 2 objectives of the course in the three areas as well as skills expected to be acquired by students are presented. Section 3 is devoted to the description of the methodology used, including an overview of the projects and information about the course organisation. The implemented system for evaluation of students progress is presented in Section 4. Finally, remarks on the experiences obtained during the last three years are presented.

\section{Objectives}

The main scientific objective of the Simulation course is an introduction to the application of numerical analysis to basic materials science and fluid dynamics problems in aerospace engineering. In particular, it is expected that after completing the course, students have:

- A theoretical knowledge of the models describing some basic aerospace problems.

- A conceptual understanding of numerical methods commonly used in the analysis of aerospace systems.

- A working knowledge of these numerical methods and experience in implementing them.

- A physical interpretation of the results derived from numerical simulations.

- A practical knowledge of visualization techniques.

- An intoductory knowledge of CFD commercial packages.

In order to accomplish these objectives in a comprehensive way, fundamentals of theoretical models and several numerical and programming techniques for computation and visualization are presented by specialists in each area. The course counts with the participation of lecturers coming from the departments of Applied Physics, Applied Mathematics IV and Computer Architecture of the Technical University of Catalonia (UPC), and are specialized in the physical description, the numerical analysis and the programming and visualization aspects, respectively. 
Besides the objectives related to scientific and technical knowledge, the course also aims at developing other skills like group work, project development, scientific documents writing, use of English and performing oral presentations.

The specific objectives of each area as well as an explanation on the other skills are presented in the following subsections.

\subsection{Physical Description}

When students enrol Simulation, they have already followed courses on Fundamental Physics, Thermodynamics, Materials Science and Aerodynamics. However, no computational tools to solve problems related with these topics have been used before.

The main goal from the physical point of view is to show how some physical phenomena related to aerospace applications in fluid dynamics and materials science can be addressed in an reachable way by means of simplified models and adequated numerical techniques. Besides this objective, it is also an important issue to promote in students a critical point of view in any numerical results they can obtain.

Projects development throughout the course share some common objetives such as the understanding of the involved physics and the models used to simulate them as well as the physical interpretation of the obtained results.

\section{$2.2 \quad$ Numerical Methods}

An introduction to the basics of numerical analysis and partial differential equations is the main objective in the area of numerical methods. The considered topics are:

- Numerical integration (the trapezoidal rule and Simpsons method, including error estimation in both cases).

- Discrete approximation of derivatives.

- Numerical methods for ordinary differential equations (from Euler's to RungeKutta method and introducing predictor-corrector methods).

- Partial Differential Equations (only second order and mainly parabolic and hyperbolic equations).

Aimed at acquiring a working knowledge in numerical methods, a brief introduction strongly facilitates the study of the discretization procedures and the numerical treatment of those partial differential equations that govern the main problems in fluid dynamics which the students will face in the course.

\subsection{Programming and Visualization}

From the programming perspective, the main goal of the course is to make students acquire a basic knowledge and understanding of Object Oriented Programming (OOP) in $\mathrm{C}++$ and the necessary skills to develop applications with a graphical user interface (GUI). It is noteworthy that previous programming 
experience of students is limited to an introductory course to $\mathrm{C}$ programming followed by another course where OOP is introduced but only addressing encapsulation. After these courses a full year passes before they can enrol Simulation.

The following list summarizes the main objectives of the course in this area:

- Introduction to GNU/Linux: during the course students will familiarize with this system at a user level.

- Object Oriented Programming in $\mathrm{C}++$ : students will have to brush up on their $\mathrm{C}++$ and learn new concepts as they work on their projects.

- Real numbers in computing: due to the numerical nature of the algorithms that will be implemented having notions on this topic will help understing some issues that may arise.

- Application programming and debugging with KDevelop: KDevelop [4 is an Integrated Development Environment that provides an editor, compilers, a debugger, etc. thus sharing features with most IDEs.

- Application progamming using Qt GUI toolkit: the Qt [5] libraries provide a quite comprehensive set of classes for GUI programming. Besides it is multiplatform, well documented, integrates well with KDevelop and provides an easy to use interface designer.

\subsection{Skills}

There are a number of important skills whose development also forms part of the course objectives:

- Group work: Most of the course work is done in group.

- Self learning: There are no in-depth explanations to the class, rather they are given introductory notions and pointers to different topics that they will need to work on.

- Report writing: One of the items that must be delivered after the project completition is a report as described in Section 3

- Performing oral presentations: Each group must give a presentation to the class explaining the main aspects of their project and demoing the developed application.

- Use of English: Unfortunately, the use of English is not integrated into the studies to a point in which students are able to use it fluently. We try to alleviate this situation by providing all course materials in English. At least, students should be comfortable reading technical documentation in English.

\section{Methodology}

In this Section the learning methodology used in the Simulation course is presented. It starts with a very brief introduction to Project Based Learning, following an overview of the materials science (training project) and CFD projects that students will have to develop. Finally, we describe how the course is organized around the project development process. 


\subsection{Project Based Learning}

The basic idea of Project Based Learning (PBL) [123] is to organize the course (or most of it) around a project which the students must work on in groups. During the development process they need to learn the necessary topics in order to carry it out.

PBL provides a model based on the student learning process rather than focusing on the lecturer teaching activities. It is a quite extended methodology, specially in engineering studies, where it fits very well. Nevertheless, it is not easy to implement because it confronts both lecturers and students with a number of challenges.

\subsection{Projects Overview}

Three consecutive projects are developed by students throughout the course. A first short training project on crystal growth, a largest CFD project, and a final project using a commercial tool. The first two projects, in which students implement their own codes, are explained in this Section.

The first project developed during the course is a training one developed by all the groups. It consists in the numerical simulation of the process of a crystal growing from an undercooled melt. A simple version of the phase-field model introduced in [6] is used to reproduce the physical phenomena. This project allows students to familiarise with finite difference techniques, implicit schemes, mesh related problems and to start implementing visualisation techniques. Different initial and boundary conditions and system sizes are considered. From the physical point of view, students acquire an idea of the role played by undercooling and anistropies on the time evolution of the system.

The projects in the fluid dynamics field are inspired in [7] and are the following:

1) Subsonic-supersonic isentropic nozzle flow.

2) Incompressible Couette flow.

3) Prandtl-Meyer expansion wave.

Each group carries out only one of the CFD projects. Students directly acquire the knowledge associated with the project that they develop, while knowledge on the other projects is acquired through the lecturer presentations and, at the end of the course, through the oral presentations of the other groups.

In project 1), the flow of a gas through a convergent-divergent nozzle is simulated. In order to simplify the model to use, the study is carried out for quasi-onedimensional flows. A set of dimensionless finite-difference equations (continuity, momentum and energy) are solved by means of MacCormack's technique for an specific nozzle shape and initial conditions. The goal of this project is to obtain the steady-state solution. Figure 1 (left) shows an example of the graphical interface developed for this project.

In project 2), incompressible Couette flow is studied as an example of a simple viscous flow that retains much of the same physical characteristics of a more complicated boundary-layer flow. The governing equations for this problem are 

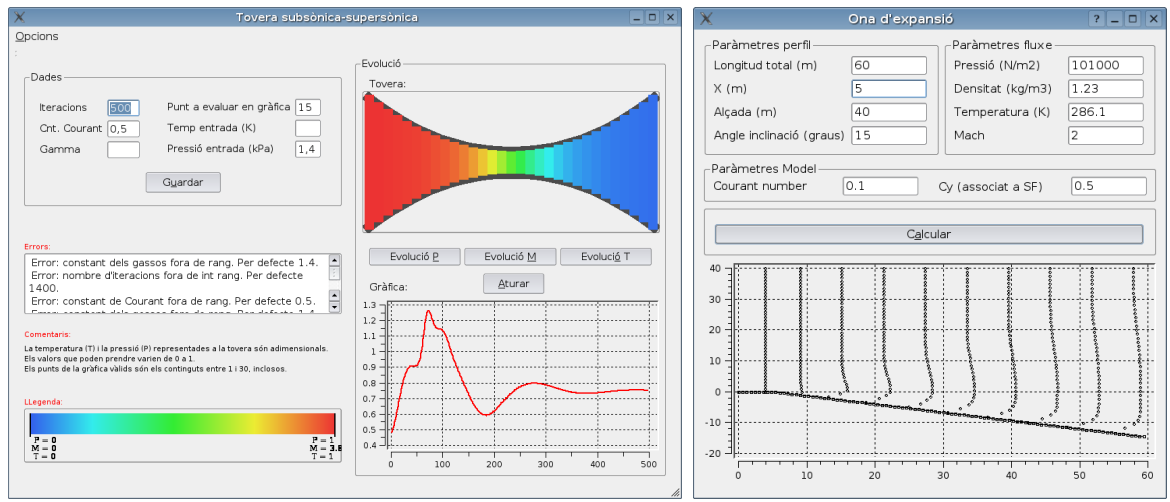

Fig. 1. Project examples. Left: subsonic-supersonic nozzle, right: Prandtl-Meyer expansion wave.

parabolic partial differential equations, while in projects 1) and 3) are hyperbolic differential equations. The employed numerical technique for the solution of the Couette flow is the Crank-Nicholson implicit method. In this case, the steady-state solution can be easily validated by the corresponding analytical solution of the problem. The observation of the evolution of the velocity profiles in an unsteady flow is an example of the added value provided by the numerical simulation.

A two-dimensional, inviscid, supersonic flow moving over a surface is studied in project 3). This problem shows an special interest when the surface contains a sharp corner. In this situation, the supersonic flow is expanded around the sharp expansion corner in such a way that an expansion wave (the Prandtl-Meyer expansion wave) made up of an infinite number of infinitely weak Mach waves, fans out from the corner. The corresponding Euler equations for the considered flow are solved by means of the MacCormack's predictor-corrector explicit finite-difference method applied to downstream space marching. An additional difficulty of this project lies in the necessity of carrying out a grid transformation to change from the physical plane to a rectangular computational plane. An example of the graphical interface developed for this project is shown in Figure 1 (right).

In general, the projects are not specially complex from a class design point of view. Most groups end up with just two to three classes involved in the simulation computation. It is in the development of the graphical user interface where students will have to deal with a hierarchically structured collection of $\mathrm{C}++$ classes that provide the different graphical components.

The interdisciplinary nature of the projects makes its development a richer experience and also more real and encouraging. Besides, its open-endness allows the more motivated and capable groups to add features beyond the required minimums.

\subsection{Course Organisation}

Simulation requires about 250 hours of workload for the students, including classroom sessions. It has a duration of 15 weeks with 7 hours of classroom 
sessions per week. There are 25 available places which, from its beginning, have always been covered by demand.

As seen on table 1, the Simulation course is organized in three blocks. The first block corresponds to the training project, which is delivered during the fourth week. Once the training project has been done, a more challenging and bigger fluid dynamics project is presented. Students will be working on this project for approximately seven weeks. Finally, the remaining four weeks of the course are devoted to the introduction and use of a widely used commercial flow modelling software. All lecturers of the course participate in both the trainig and the main project. The remainder of the course is done by only one of the lecturers.

Table 1. Course organisation

\begin{tabular}{llll}
\hline Block & Duration & Deliverables & Lecturers \\
\hline Training Project & 4 weeks & Working application & Physics, Math, Comp \\
Main Project & 7 weeks & $\begin{array}{l}\text { Application, writen } \\
\text { report and oral }\end{array}$ & Physics, Math, Comp \\
& & $\begin{array}{l}\text { presentation } \\
\text { Tutorials, writen re- Physics } \\
\text { port and working ap- } \\
\text { plication }\end{array}$ \\
\hline
\end{tabular}

At the beginning of the course students are organized in groups of 3 to 4 members. During the first weeks the main topics that will be covered during the course are introduced. The training project is proposed at the same time. This project aims at making students get familiar with the basic concepts and the tools that will be used during the course. It also forces students to get rapidly started and helps in establishing group work dynamics.

During the second block of the course students carry out the development of the main project. First all projects are presented, then the course coordinator negotiates the distribution of the projects among the different groups. Since the difficulty level may differ from project to project, the results obtained in the training project will be helpful in order to decide on this distribution. From that point on, students work on their own. During the development process students will face different physics, mathematical and programming problems that they will need to work on in order to make progress. Thus the study of the different topics of the course is prompted by the learning needs that stem from the project development. Two books of J.D. Anderson [78] are the basic reference materials of the course, providing guidance and sample results that must be matched by the simulation programs outcome. This phase ends with the delivery of three different items:

1. The application that simulates the fluid dynamics problem.

2. A written document containing:

(a) an abstract both in English and Spanish or Catalan, 
(b) a description of the physical problem,

(c) its mathematical resolution,

(d) and the main aspects of the program implementation with a minimal user manual.

3. A 20 minutes oral presentation, summarizing the information contained in the document, with a demo of the application.

During the final weeks of the year, students are introduced to Gambit, the geometry and mesh generation tool of the Fluent CFD commercial package 9]. This block of the course is done by the lecturer from the physics department. At this point students already have a basic understanding of numerical simulation principles which facilitates the use of commercial tools. After some introductory lectures, students carry out six tutorials provided by Fluent in order to practise the basic points of geometry creation and mesh generation and refinement. The final part of this block is devoted to the development of a project using Gambit. Under the lecturer advise, students choose an aerospace system (e.g. wings, balloons) and generate its geometry and create and study different meshes. Students have the possibility to complete this work in the Computational Fluid Dynamics course offered in the following semester. In this other course, the Fluent solver is introduced and students can use it to study the behavior of fluids flowing in the geometries built in the project done in Simulation.

An important point of the methodology is the role of the lecturers, that gradually changes as the course advances. During the first weeks the lecturing activity is rather based on talks about the different topics that must be introduced. Once students start working on their projects their activity will consist in solving questions, supervise students progress and, from time to time, give small clarifying lectures if deemed necessary. During scheduled times at the classroom students will be able to perform their group meetings, work on their projects and ask whatever questions they have.

Finally, in order to facilitate students work, the Castelldefels School of Technology provides every group of the Simulation course with a laptop for their use during the whole semester. The laptop comes with all the necessary software pre-installed. Students can also take advantadge of the wifi network available in most areas of the campus. Additional computers are available at the room where sessions take place. This is a key point which facilitates meetings and work in group.

\section{Assesment}

There is a separate evaluation of the activities done by students throughout the different blocks of the course. The whole part of the evaluation process is done by the evaluation comission, composed by the three lecturers in charge of this course.

The first part of this evaluation comes from the document of the training project delivered by students. Grades are determined by the quality of the project and represent $10 \%$ of the final grading. 
At the seventh week of the year, students take the mid-term exam which lasts 90 minutes. General or specific questions about the introduced concepts on the physics, numerical or visualisation part are posed. Grades obtained in this exam represent $20 \%$ of the total grading of the course.

The CFD project is evaluated from the document presented as well as from the public presentation done by groups. In order to pass this evaluation, students have to present some predetermined results which basically serve to validate their simulations. Students are encouraged to perform additional studies in their project in order to get higher marks. This part represents $50 \%$ of the final grading.

Work performed in the last block of the course is evaluated through the delivery of a short document on the project of geometry construction and meshing with Gambit and represnets $10 \%$ of the total grading of the course.

Finally, a $10 \%$ of the grading comes from the subjective evaluation done by the commission on the students participation in each activity.

\section{Concluding Remarks}

In this article the implementation of the Simulation course offered at the Technical School of Castelldefels (EPSC) has been discussed. The two main innovative aspects of the course are the use of PBL as the learning methodology and the participation of lecturers from different areas of expertise. The course is being run since 2004 and the experience gathered during its past three editions lets us draw some conclusions.

Due to the interdisciplinary nature of the course the participation of lecturers from differenet areas of expertise fits quite naturally. This is uncommon in the Spanish university context where each course is usually assigned to a single department. We believe that this is good for both lecturers and students. Lecturers become more familiar with the activities carried out by their colleagues and students are provided with a broader view of the different course topics. This aspect is also positively valued by students. The main challenge that must be addressed is an adequate coordination of lecturers, so that students face a steady and manageable workload. Also care needs to be taken in order to provide students with a common view regarding all aspects of the course.

The PBL approach clearly makes students more engaged with the course. Being confronted with a challenging and realistic project is encouraging, they percieve their learning as a direct consequence of their personal effort and, at the end of the course, they feel more satisfied. As a consequence of working in a "real" project, students also think that acquired knowledge may be useful in their academical or professional future.

In respect to the use of Linux and other free and open-source software there are some complains mostly regarding the availability of Linux at home (not all students are willing to install a different OS on their personal computers). Besides having a laptop available at all times, this issue has been greatly alleviated by virtualization tools such as VirtualBox [10] and the possibility of dual booting. 
The availability of finalized projects from previous editions and students doing little work within their group could be matters of concern. The continuous supervision of groups work at the classroom makes both issues unlikely to pass unpercieved and these situations have not been detected yet. A concern that we are studying how to address is an excessive degree of specialization within the groups. As an example, programming is not equally done among the group members. This can be seen during the classroom sessions and is confirmed by the exam results. Also, sometimes we feel that differences among students of a given group may not be properly reflexed in the final marks.

To conclude, we are satisfied with the quality of most delivered projects and with the course results in general. It is also worth noting that the studentcentered active learning approach applied in the course suits perfectly to the methodologies shift that should accompany the adaptation process to the new European framework.

\section{References}

1. Markham, T.: Project Based Learning, a guide to Standard-focused project based learning for middle and high school teachers. Buck Institute for Education (2003)

2. Crawford, A., Tennant, J.: A Guide to Learning Engineering Through Projects. University of Nottingham (2003)

3. Del Canto, P., Gallego, I., Hidalgo, R., López, J., López, J.M., Mora, J., Rodríguez, E., Santamaria, E., Valero, M.: Aprender a Programar Ordenadores mediante una Metodología Basada en Proyectos. $18^{\circ}$ Congreso Universitario de Innovación Educativa en las Enseñanzas Técnicas (2007)

4. KDevelop Integrated Development Environment, http://www.kdevelop.org/

5. Qt GUI Toolkit, http://trolltech.com/products/qt

6. Wang, S.-L., Sekerka, R.F., Wheeler, A.A., Murray, B.T., Coriell, S.R., Braun, R.J., McFadden, G.B.: Physica D, 69 (1993)

7. Anderson Jr., J.D.: Computational Fluid Dynamics. The basics with applications. McGraw-Hill, New York (1995)

8. Anderson Jr., J.D.: Fundamentals of Aerodynamics. McGraw-Hill, New York (2001)

9. http://www.fluent.com/

10. VirtualBox, http://www.virtualbox.org/ 